\title{
1 Antarctic biodiversity predictions through substrate qualities and \\ 2 environmental DNA
}

3 Authors:

4 Paul Czechowski ${ }^{1}$, Michel de Lange ${ }^{2,3}$, Micheal Knapp ${ }^{1}$, Aleks Terauds ${ }^{4}$, and Mark I.

5 Stevens 5,6

6 Affiliations:

$7{ }^{1}$ University of Otago, Department of Anatomy, 270 Great King Street, Dunedin, OTA 9016,

8 New Zealand.

$9{ }^{2}$ University of Otago, Department of Biostatistics, 18 Frederick Street, Dunedin, OTA 9016,

10 New Zealand.

$11{ }^{3}$ Pacific Edge Ltd, Centre for Innovation, 87 St David Street, Dunedin, OTA 9016, New

12 Zealand.

$13{ }^{4}$ Australian Antarctic Division, Department of Agriculture, Water and the Environment, 203

14 Channel Highway, Kingston, TAS 7050, Australia.

$15 \quad{ }^{5}$ South Australian Museum, 61-68 North Terrace, Adelaide, SA 5000, Australia.

$16{ }^{6}$ School of Biological Sciences, The University of Adelaide, SA 5005, Australia.

17 *Correspondence to Paul Czechowski, paul.czechowski@otago.ac.nz

18 Open Research Statement:

19 Parts of the data are already published, with those publications cited in this article. All data

20 were provided as in-confidence for peer review and have been revised during peer review to

21 accompany this article. All versions are available via https://doi.org/10.5281/zenodo.4579841

22 and github.com/OldMortality/eukaryotes.

\section{Abstract:}

24 Antarctic conservation science is important to enhance Antarctic policy and to understand 25 alterations of terrestrial Antarctic biodiversity. Antarctic conservation will have limited long26 term effect in the absence of large-scale biodiversity data, but if such data were available, it is 27 likely to improve environmental protection regimes. To enable Antarctic biodiversity 28 prediction across continental spatial scales through proxy variables, in the absence of baseline 29 surveys, we link Antarctic substrate-derived environmental DNA (eDNA) sequence data 
30 from the remote Antarctic Prince Charles Mountains to a selected range of concomitantly

31 collected measurements of substrate properties. We achieve this using a statistical method

32 commonly used in machine learning. We find neutral substrate $\mathrm{pH}$, low conductivity, and

33 some substrate minerals to be important predictors of presence for basidiomycetes,

34 chlorophytes, ciliophorans, nematodes, or tardigrades. Our bootstrapped regression reveals

35 how variations of the identified substrate parameters influence probabilities of detecting

36 eukaryote phyla across vast and remote areas of Antarctica. We believe that our work may

37 improve future taxon distribution modelling and aid targeting logistically challenging

38 biodiversity surveys.

\section{Introduction:}

40 Although only $0.3 \%$ of continental Antarctica is ice-free, many organisms including bacteria,

41 unicellular eukaryotes, fungi, lichen, cryptogamic plants and invertebrates are scattered

42 across the continent in extremely isolated, remote, island-like terrestrial habitats, for example

43 in soil-like substrates, lakes, and cryoconite holes (Convey et al. 2014; Chown et al. 2015).

44 Threats to this Antarctic biodiversity are posed by human activity, climate change, pollution,

45 and invasive species. It is becoming increasingly clear that mitigation of these threats and

46 further alterations to the Antarctic biosphere rely on well-tailored management strategies

47 across the continent's bioregions (eg Coetzee et al. 2017).

48 Effective continental-scale conservation management requires continental-scale data (eg

49 Wauchope et al. 2019). However, knowledge of terrestrial Antarctic biodiversity is still

50 limited because most of Antarctica's ice-free areas remain unstudied due to logistic

51 difficulties exacerbated by the harsh environmental conditions, and funding constraints.

52 Environmental DNA (eDNA) analysis, despite shortcomings, is arguably one of the most

53 practical and economical options for continental-wide baseline surveys of terrestrial Antarctic

54 biodiversity, especially when facing logistical challenges typical for work on the Antarctic

55 continent (reviewed in Czechowski et al. 2017). Comparable large-scale systematic

56 approaches to protect soil diversity are recognized as required globally, but often are limited

57 to charismatic groups such as those found in the Arctic (Gillespie et al. 2020).

58 Here, we link commonly measured substrate properties to the cryptic eukaryotic biodiversity

59 of terrestrial Antarctic ice-free regions. Soil nutrient status is the most important attribute of

60 biodiverse soils (Geisen et al. 2019), and corresponding key variables can be, and are,

61 routinely measured economically. We analyzed molecular data (eDNA) from an extremely 
62 remote Antarctic terrestrial region to clarify relationships between substrate properties and

63 eukaryote phylum presence. We envisage our approach to be useful in predicting biodiversity

64 across a wide taxonomic spectrum across large areas of Antarctica, especially to identify

65 regions worthy of lower-level taxonomic biodiversity surveys, then possibly realized with

66 "barcoding" using mitochondrial DNA (such as with the Cytochrome Oxidase 1) or

67 logistically more challenging morphological biodiversity assessments.

68 The Prince Charles Mountains (PCMs), the most remote terrestrial areas in eastern

69 Antarctica, were first sighted by US Operation Highjump (1946/47) and mapped in more

70 detail by Australian (1954-1961) and Russian (1983-1991) expeditioners. In 2011 we

71 obtained environmental DNA samples from substrates throughout the PCMs and measured

72 various geochemical and mineral properties. Previously, Czechowski et al. (2016b) focused

73 on invertebrates as the primary substrate-inhabiting metazoans and discovered major changes

74 in their distribution over salinity gradients, as known from other areas and taxa of Antarctica

75 (eg Bottos et al. 2020). Here, we expand our analyses of environmental variables using a

76 predictive approach to the full spectrum of eukaryote phyla, and thereby explore approaches

77 of inferring biodiversity presence that could be applied across the entirety of ice-free

78 terrestrial Antarctica. Beyond phylum-level surveys, our technique may be applied using

79 other genetic markers and predictors to link future smaller-scaled conservation projects

80 anywhere in terrestrial Antarctica, aid taxon distribution modelling, and thus contributes

81 towards improving conservation management strategies across the Antarctic bioregions.

\section{Methods:}

83 Fieldwork took place in the Prince Charles Mountains (PCMs; East Antarctica, Figure 1)

84 from 26 November 2011 to 21 January 2012 close to Mount Menzies (MM; 73²5'29.38"S, $8562^{\circ} 0^{\prime} 37.61^{\prime \prime E}$ ), Mawson Escarpment (ME; 7319'16.91"S, 68 19'31.20"E) and Lake 86 Terrasovoje (LT; 70³2'23.58"S, 6757'28.05"E) as described earlier (Czechowski et al. 87 2016a, b). 154 field samples were considered for this study (26 MM, 70 ME, 58 LT; Web 88 Table 1).

89 To infer climatic conditions in the PCMs, we used rasters from Quantarctica 3 (Matsuoka et 90 al. 2021) encoding annual mean precipitation ( $\mathrm{mm})$, wind speed ( $\mathrm{m} \mathrm{s}^{-1} 10 \mathrm{~m}$ above ground) 91 and mean annual temperature $\left({ }^{\circ} \mathrm{C} 2 \mathrm{~m}\right.$ above ground, as only temperature data distributed via 92 Quantarctica). We disaggregated the layer rasterization from $35 \mathrm{~km} \mathrm{px}^{-1}$ to $1 \mathrm{~km} \mathrm{px}^{-1}$ through 
93 bilinear interpolation. We then extracted median values for the three variables from a $20 \mathrm{~km}$

94 buffer surrounding each sampling location (Web Figure 1).

95 As predictor data for eukaryote phylum presence in substrates, geochemical composition

$96\left(\mathrm{NH}_{4}{ }^{+}, \mathrm{C}, \rho, \mathrm{NO}_{3}{ }^{-}, \mathrm{pH}_{\mathrm{H} 20}, \mathrm{pH}_{\mathrm{CaCl} 2}, \mathrm{P}, \mathrm{K}, \mathrm{S}\right.$, texture $)$ was analyzed by agricultural soil testing

97 service APAL (www.apal.com.au). Many measurements below detection level needed to be

98 excluded to yield data completeness of at least $96.7 \%$ (Web Table 2). The final analysis

99 included $\mathrm{K}, \mathrm{S}, \rho$, and $\mathrm{pH}_{\mathrm{CaCl} 2}\left(\mathrm{pH}_{\mathrm{H} 2 \mathrm{O}}\right.$ excluded as co-linear, texture excluded as categorical).

100 As additional predictors, the substrate mineral composition was considered through

101 integration of X-ray diffraction spectra of the minerals quartz, calcite, feldspar, titanite,

102 pyroxene / amphibole / garnet, micas, dolomite and kaolin / chlorite, and chlorite (see

103 Czechowski et al. 2016b).We handled the sum-to-unity constraint of our mineral

104 compositions by excluding quartz as the most common mineral from further analysis. As

105 further predictors for most locations (MM: n=26, ME: n=69, LT: $n=57$ ), we included hitherto

106 unpublished measurements of soil-substrate ATP (eg Conklin and Macgregor 1972), obtained

107 with a Clean-Trace Luminometer (3M, Maplewood, US-MN), and slope measurements. Prior

108 to regression, all predictors were standardized to mean of 0 and unit variance. Predictor

109 densities are provided in Web Figure 2.

110 Biological response data were prepared in QIIME 2020-2 (Bolyen et al. 2019) and R 4.0.0 (R

111 Core Development Team 2019) from raw sequence data generated as described elsewhere

112 (Czechowski et al. 2016b, 2017). In summary, 125 bp eukaryotic 18S rDNA PCR products

113 (yielding an 85 bp target region) had been amplified using primers 'Euk1391f' and 'EukBr'

114 (Caporaso et al. 2012), as established for eukaryotic microbial surveying (Thompson et al.

115 2017). As recommended, PCRs had been carried out in triplicates, each replicate carrying

116 identical barcodes. The resulting eDNA libraries had been combined for sequencing across

117 two MiSeq runs (Web Figure 3). We re-defined Amplicon Sequence Variants (ASVs; sensu

118 Callahan et al. 2017) from those data with Qiime: after pre-filtering (Phred score $\geq 25$ ), we

119 trimmed read pairs with Cutadapt v1.18 (Martin 2011), and denoised using DADA2 (v1.6.0;

120 Callahan et al. 2016). We retained merged reads with an expected error value less than 3, that

121 we not deemed chimeric.

122 Due to the shortness and slow evolution of the employed 18S marker, we set out to conduct

123 our analyses on the phylum level, and to use species level assignments solely to verify data

124 credibility. Accordingly, we designed the retrieval of taxonomic annotations for our Antarctic

125 DNA sequences in such fashion so as to yield reliable species identifications in cases where 
126 Antarctic reference data were available, while still retuning higher taxonomic (eg phylum

127 level) identifications in cases where closely matching reference data were not available.

128 Doing so, we were able include a larger amount of Antarctic sequences into our statistical

129 analysis on phylum level, but needed to consider species level identifications as potentially

130 unreliable, and verify them on alignment level. We identified eukaryotic sequences among

131 our reads with a recent local copy (April 2020) of the entire NCBI nucleotide collection in

132 conjunction with Blast 2.10.0+. Taxonomic assignments were retrieved from reference

133 sequences at least 50\% identical to queries, with an assignment significance threshold (e

134 value) of $10^{-10}$, considering only matches with at least $90 \%$ coverage, and excluding

135 environmental sequences (evalue $1 e^{-10}$, max_hsps 5, max_target_seqs 5, qcov_hsp_perc 90

136 and perc_identity 50). For each Antarctic sequence search query, we used the highest Bit

137 score among all returned sequences from the NCBI database for that query to choose the final

138 taxonomic assignment. Subsequently, we used R package decontam (Davis et al. 2018) to

139 remove putatively contaminating reads, and likewise subtracted all sequences and taxa in

140 negative controls from field samples. Focusing on eukaryotes, we discarded all non-

141 eukaryote reads (Web Figure 4).

142 With the Lasso (Tibshirani 1996) of R package glmnet (Friedman et al. 2010) we regressed 143 each phylum present in at least 12 samples against the aforementioned predictors (Web 144 Figure 5). In regressions, we disregarded sequence read abundances as meaningless due to 145 inherent constraints of amplicon sequencing (eg Czechowski et al. 2017), analyzed presences 146 instead, and used the most biodiverse of all locations (LT; Czechowski et al. 2016b; also 147 Figure 2) as a reference location, so that we report predictor effects at MM and ME as 148 relative to LT. We initially retrieved the active set (variables not set to 0 ) estimated by Lasso, 149 repeated the regression of phylum presence against 1,000 randomly chosen sample-sets of 150 predictors, calculated the number of times each variable was estimated to be non-zero, and 151 report variables non-zero more than 950 times as significant. Accordingly, we calculated $15295 \%$ non-parametric bootstrap confidence intervals for our estimates. We did not adjust for 153 multiple comparisons.

154 Furthermore, we explored the global distribution of the obtained putative species level 155 assignments among phyla significantly influenced by environmental predictors (see below) 156 by querying BISON (bison.usgs.gov), GBIF (www.gbif.org) and iNaturalist 157 (www.inaturalist.org; see Web Text 1 for detailed methods) with R package spocc.

\section{Results:}


159 Keeping in mind the coarse raster resolution and model-like character of the climate data, 160 annual mean climate at MM was coldest $\left(-32 \pm 0.3{ }^{\circ} \mathrm{C}\right)$, windiest $\left(10.2 \pm 0.05 \mathrm{~ms}^{-1}\right)$ and with 161 an intermediate amount of precipitation $(86 \pm 1 \mathrm{~mm})$, when compared to the other two 162 locations (Web Figure 1). ME exhibited the least amount of precipitation $(55.3 \pm 7 \mathrm{~mm}$ ), 163 comparatively low wind speeds $\left(5.4 \pm 0.5 \mathrm{~ms}^{-1}\right)$, and slightly higher temperatures than MM ($16428.4 \pm 0.6 \mathrm{~mm}$ ). Closest to the coast, and exposed, LT appeared influenced by the highest 165 precipitation $(136 \pm 16 \mathrm{~mm})$, variable but moderate wind speeds $\left(5.5 \pm 1.7 \mathrm{~ms}^{-1}\right)$ and the 166 highest temperature in the surveyed area $\left(-24.1 \pm 1.6{ }^{\circ} \mathrm{C}\right)$. We found our chosen climatic 167 variables strongly correlated with the sampling locations, and to improve predictive power 168 excluded the former from further considerations. Instead, we interpreted the statistical effect of location (below) to be a function of annual mean climatic variables.

170 Retention of eukaryotes in field-derived samples after filtering yielded 2,285,773 reads across 171145 samples, derived from 16,524,031 unfiltered sequences (Web Table 3). Per-sample mean 172 coverage was 9,450 reads (min: 2, median: 2,379, max: 86,804). ASV mean coverage after 173 filtering was 2,984 reads (min: 2, median: 132, max: 207,718; Web Figure 6). Collectively 174 after filtering, 766 ASVs were assigned to 495 species across 25 phyla (Web Table 4). Most 175 prevalent phyla (and among those: most prevalent species) by coverage were Ascomycota 176 (Acanthothecis fontana), Chlorophyta (Coccomyxa sp.), Basidiomycota (Mrakia frigida), 177 Ciliophora (Pseudochilodonopsis quadrivacuolata), Nematoda (Scottnema lindsayae), 178 Rotifera (Embata laticeps), and Tardigrada (Mesobiotus furciger). (All taxonomic 179 assignments listed here aligned with reference data without gaps at full coverage, and a bit 180 score of 154.6, apart from bit score of 145.6 for P. quadrivacuolata)

181 We found the distribution of five phyla (26 classes, 59 orders, 100 families, 173 species) 182 across the PCMs to be significantly correlated with the considered soil predictors (Figure 2, 183 Web Tables 5 and 6, Web Figure 7). Those taxa were defined by 265 ASVs across 1,210,855 184 sequences and 142 samples (23 MM, 64 ME, 55 LT). Per-sample mean coverage was 9,460 185 (min: 2, med: 3863, max: 84,892), per-ASV mean coverage was 4,596, (min: 2, median: 157, 186 max: 128,358; Web Figure 6).

187 For each predictor significantly correlating with a phylum's presence (Web Figure 8) we 188 report the expected effect on phylum presence corresponding to one standard deviation $(\sigma)$ 189 increase of the predictor from its mean $(\mu)$, with all other variables held at mean $\mu$. Key 190 significant results included: 
i) Low levels of Basidiomycota (62 putative species assignments, Figure 2a) in high $\mathrm{pH}$ environments $(\mu=7.15, \sigma=0.88$, E[present $\mu]=0.6$ and $\mathrm{E}[$ present $\mu+1 \sigma]=0.4)$, and a strong positive relationship of this phylum with dolomite $(\mu=0.025 \%, \sigma=0.05 \%$,

ii) Very low levels of Chlorophytes (47 species, Figure 2b) at MM plausibly attributable E[present $\mu+1 \sigma]=0.7)$.

iii) Very low levels of Ciliophorans (47 species, Figure 2c) at MM (E[present $\left.{ }_{L T}\right]=0.70$

iv) Very low levels of nematodes (8 species, Figure $2 \mathrm{~d}$ ) at $\mathrm{MM}\left(\mathrm{E}\left[\right.\right.$ present $\left._{\mathrm{LT}}\right]=0.47$ and $\mathrm{E}\left[\right.$ present $\left.\left.{ }_{\mathrm{MM}}\right]=0.28\right)$, and in highly conductive substrates $\left(\mu=0.55 \mathrm{dSm}^{-1}, \sigma=1.07\right.$

v) Very low levels of tardigrades (9 species, Figure 2e) in alkaline substrates (E[present $\mathrm{dSm}^{-1}, \mathrm{E}\left[\right.$ present $\left._{\mu+1 \sigma}\right]=0.35$ )

212 Observed fractions of non-zero coefficients are shown Table 1 and Web Figure 8. (95\% non-

213 parametric bootstrap confidence intervals for non-0 estimates also provided in Web Figure 8.)

214 Directions of all predictor effects on all analyzed taxa presences, including insignificant 215 effects, are listed in Web Table 7.

216 For 66 of our 173 putative species assignments 778 georeferenced records could be obtained 217 (of those 65\% from GBIF, 27\% iNaturalist, 7\% BISON). Of the obtained 123 locations 4\% 218 were in Africa, 1.6\% in Antarctica, 13\% Asia, 32\% Europe, 21\% North America and 10\% in 219 South America (Web Figures 9 and 10, Web Table 7). The sole species recorded for 220 Antarctica (here: south of $66.56^{\circ}$ ) was the nematode Scottnema lindsayae. Observations north 221 of the polar circle (likewise $66.56^{\circ}$ ) included Basidiomycota (Gloiocephala aquatica, 222 Stereum rugosum, Mrakia frigida, Rhodotorula mucilaginosa), Chlorophyta (Haematococcus 223 lacustris, Oophila amblystomatis), and Ciliophora (Furgasonia blochmanni, Chilodonella 
224 acuta (Ciliophora), Tachysoma pellionellum). Refer to Web Tables 4 and 5 for alignment

225 qualities.

\section{Discussion:}

227 Our Antarctic case study demonstrates two key technologies to be useful for baseline

228 biodiversity surveys across large spatial scales in extremely remote environments - robust

229 predictive statistics, such as the Lasso, now often used in machine learning algorithms

230 (Muthukrishnan and Rohini 2016), as well as biodiversity information derived from

231 environmental DNA (Czechowski et al. 2017). To the best of our knowledge, our work is the

232 first in associating environmental DNA data to environmental predictors by means of the

233 Lasso to yield accurate detection probabilities for taxonomic groups, also in Antarctica. Thus,

234 we present an analytical framework to identify areas for targeted species-level biodiversity

235 surveys, using other markers, or predictors for Antarctica, and possible other hardly

236 accessible locations.

237 Our expanded analyses of the original raw data (Czechowski et al. 2016b) made use of new 238 algorithms for processing environmental DNA sequences (eg Callahan et al. 2016, 2017),

239 along with more extensive reference databases for taxonomic assignment, and new

240 algorithms available with R (R Core Development Team 2019). While our results are in line

241 with earlier findings relating eukaryote distribution to their environment in the PCMs and

242 Antarctica (eg Czechowski et al. 2016a, b; Bottos et al. 2020), our approach adds accuracy to

243 those findings with respect to five phyla.

244 A strength of our analyses is the relatively easy retrieval of biological survey data

245 encompassing many phyla (probably including many cryptic and unknown species) across

246 many samples. The weakness of the employed 18S marker is its limited ability to discern

247 many distinct sequence variants on a low taxonomic (eg species) level. Regardless,

248 identification of species with likely Antarctic occurrence such as the known Antarctic

249 nematode Scottnema lindsayae and tardigrade Mesobiotus furciger by means of a relatively

250 short and highly conserved primer pair highlights the ability of environmental DNA to

251 retrieve species occurrence records, provided that sufficient sequence data is available for

252 taxonomic assignment. Consequently, we believe that environmental DNA analysis should be

253 the method of choice to obtain biodiversity data from Antarctica, particularly when many

254 samples are to be analyzed, but other markers are needed to investigate fine scaled

255 endemism, and to obtain better taxonomic resolution. 
256 Georeferencing our putative species assignments by means of publicly accessible databases

257 had limited success. The limitations of reference databases became obvious when known 258 Antarctic species, such as Acutuncus antarcticus (Web text 1), the latter identified among our 259 data through a perfect alignment with bit score 154.6, were not found, and only $38 \%$ of all 260 putative Antarctic species assigned by us were georeferenced at all. High occurrence 261 prevalence in North America, and Europe indicates sampling bias in GBIF, iNaturalist and 262 BISON and highlight a substantial weaknesses of publicly accessible global biodiversity data 263 concerning cryptic eukaryotes.

264 Eukaryotic distribution patterns reported in related Antarctic studies provide context for our 265 observations from the PCMs. The rarity of Chlorophytes, Ciliophorans, and the otherwise 266 ubiquitous nematodes at MM in relation to the two other lower altitude and more northerly 267 locations (ME, LT) seem to confirm trends of increasing eukaryotic richness and diversity 268 with decreasing latitude and altitude (Czechowski et al. 2016a; Thompson et al. 2020; Zhang 269 et al. 2020), but such patterns are not always evident at the scales investigated here. Rather, 270 Antarctic biodiversity can be surprisingly regionalized (Convey et al. 2014) and 271 correspondingly, our study finds surprisingly high eukaryotic diversity to unexpectedly occur 272 even in the harshest environments, such as local ice-soil substrate boundaries at Mount 273 Menzies (Figures 1a, 2). The absence of ciliophorans from Sulphur-rich substrates, and of 274 nematodes from highly conductive soil interstices matches findings of distribution patterns 275 being shaped by age-related salt accumulation at the surface-air interface of frozen soils 276 described with other analytical approaches (Velasco-Castrillón et al. 2014b; Lee et al. 2019).

277 In absence of other predictors, our study highlights the importance of neutral substrate $\mathrm{pH}$, 278 low conductivity, and key minerals (dolomite, pyroxene, amphibole, or garnet) to predict 279 high eukaryote density in Antarctic substrates. We corroborate the negative influence of 280 substrate alkalinity on Antarctic Basidiomycota (Arenz and Blanchette 2011). 281 Bioregionalization notwithstanding, distance to coast once more appears as suitable proxy 282 variable negatively related to the presence of chlorophytes and ciliophorans (Thompson et al. 283 2020). Additionally, we find soil alkalinity, Sulphur content and substrates pyroxene, 284 amphibole, or garnets to constrain distribution of the former. Among nematodes, our results 285 (i.e. perfect alignment between our Antarctic 18S sequence from Mount Menzies and an 286 annotated reference sequence) indicate that Scottnema lindsayae could likely occur in high 287 altitude and high latitude environments such as MM, but then would be influenced by the 288 species' general indifference (rather than affinity, compare Zawierucha et al. 2019) to 
289 alkaline substrates, and must be highly localized (at least at MM) if encountered at high 290 abundance (Smykla et al. 2018; Zawierucha et al. 2019). Lastly, we confirm the negative 291 association between tardigrade occurrence and alkaline substrates observed in Victoria Land 292 (eg Smykla et al. 2018).

293 Based on our findings, ice-free areas with high annual mean precipitation, low wind speeds 294 and relatively high temperatures, exhibiting substrates with a neutral $\mathrm{pH}$ and low 295 conductivity, which are rich in dolomite but poor in pyroxene, amphibole, or garnets, are 296 likely to be highly biodiverse in the Antarctic and should harbor candidates for more focused 297 conservation management and higher resolution DNA markers with morphological species 298 level investigations. Furthermore, locations with more extreme environmental conditions may 299 harbor endemic relic fauna equally warranting protection (Convey et al. 2014). Our results 300 are in line with observations in other (including polar and alpine) ecosystems, where soil $\mathrm{pH}$ 301 was found to be an important factor determining bacterial and fungal community (Siciliano $e t$ 302 al. 2014; Bottos et al. 2020). At the same time, Antarctic soil ecosystems are relatively 303 simple and are assumed to mostly lack complex biotic interactions, although such interactions 304 may be more present in coastal terrestrial ecosystems (Velasco-Castrillón et al. 2014b; Lee et 305 al. 2019). Consequently, the soil eukaryote distribution patterns observed especially at Mount 306 Menzies are likely predominantly shaped by abiotic factors and would be gradually more 307 influenced by limited biotic interactions, lower latitude substrates or more costal substrates 308 (ME, LT).

\section{Conclusion:}

310 We provide a case study highlighting the utility of environmental molecular data and 311 predictive analysis algorithms to inform on the presence of eukaryote taxa by means of 312 relatively easily measured soil predictors, which can be combined with readily available 313 climate data. Rather than recognizing trends, our analytical technique provides accurate 314 detection probabilities for Basidiomycota, Chlorophytes, nematodes, and tardigrades in 315 relation to bedrock mineral composition, $\mathrm{pH}$, conductivity, Sulphur contents, and arguably, 316 overall harshness of environmental conditions. These, here quantified, relationships enable 317 more precise distribution modeling of phylum presences over large spatial scales. Our 318 approach may be used identify regions worthy of species level biodiversity surveys, possibly 319 employing faster evolving molecular markers or logistically more challenging morphologic 320 biodiversity assessments. We believe our approach to be valuable to inform further 321 development and understanding of both Antarctic biogeography and conservation areas. 


\section{Acknowledgments:}

323 P.C. was supported by The University of Adelaide through an International Post-Graduate

324 Research Scholarship, through the Royal Society of South Australia, and the University of

325 Otago. M.S. received AAS funding from The Australian Antarctic Division, science project

3262355 and A.T. was supported by AAS 4296. Alan Cooper and M.S. received funding for this

327 project through Australian Research Council linkage grant LP0991985. M.S. and P.C.

328 received funding for this project from the Sir Mark Mitchell Foundation and the University of

329 Otago. M.K was funded by the University of Otago. We thank Robert McPhee for the artistic

330 contribution of Figure 2. We thank Diana Wall for providing helpful comments on an earlier

331 draft of the manuscript. Authors' contributions to this article conform with the CASRAI

332 Contributor Roles Taxonomy (https://casrai.org/credit).

\section{References:}

334 Arenz BE and Blanchette RA. 2011. Distribution and abundance of soil fungi in Antarctica at 335 sites on the Peninsula, Ross Sea Region and McMurdo Dry Valleys. Soil Biol Biochem $336 \quad$ 43: $308-15$.

337 Bolyen E, Rideout JR, Dillon MR, et al. 2019. Reproducible, interactive, scalable and 338 extensible microbiome data science using QIIME 2. Nat Biotechnol 37: 852-7.

339 Bottos EM, Laughlin DC, Herbold CW, et al. 2020. Abiotic factors influence patterns of 340 bacterial diversity and community composition in the Dry Valleys of Antarctica. FEMS $341 \quad$ Microbiol Ecol 96: 1-12.

342 Callahan BJ, McMurdie PJ, and Holmes SP. 2017. Exact sequence variants should replace 343 operational taxonomic units in marker-gene data analysis. ISME J 11: 113597.

344 Callahan BJ, McMurdie PJ, Rosen MJ, et al. 2016. DADA2: High-resolution sample 345 inference from Illumina amplicon data. Nat Methods 13: 581-3.

346 Caporaso JG, Lauber CL, Walters WA, et al. 2012. Ultra-high-throughput microbial 347 community analysis on the Illumina HiSeq and MiSeq platforms. ISME J 6: 1621-4.

348 Chown SL, Clarke A, Fraser CI, et al. 2015. The changing form of Antarctic biodiversity. $349 \quad$ Nature 522: 431-8.

350 Coetzee BWT, Convey P, and Chown SL. 2017. Expanding the protected area network in $351 \quad$ Antarctica is urgent and readily achievable. Conserv Lett 10: 670-80. 
352 Conklin AR and Macgregor AN. 1972. Soil adenosine triphosphate: Extraction, recovery and 353 half-life. Bull Environ Contam Toxicol 7: 296-300.

354 Convey P, Chown SL, Clarke A, et al. 2014. The spatial structure of Antarctic biodiversity. $355 \quad$ Ecol Monogr 84: 203-44.

356 Czechowski P, Clarke LJ, Breen J, et al. 2016a. Antarctic eukaryotic soil diversity of the 357 Prince Charles Mountains revealed by high-throughput sequencing. Soil Biol Biochem $358 \quad 95: 112-21$.

359 Czechowski P, Clarke LJ, Cooper A, and Stevens MI. 2017. A primer to metabarcoding 360 surveys of Antarctic terrestrial biodiversity. Antarct Sci 29: 3-15.

361 Czechowski P, White D, Clarke L, et al. 2016b. Age-related environmental gradients 362 influence invertebrate distribution in the Prince Charles Mountains, East Antarctica. $R$ $363 \quad$ Soc Open Sci 3: 160296.

364 Darienko T, Lukešová A, and Pröschold T. 2018. The polyphasic approach revealed new 365 species of Chloroidium (Trebouxiophyceae, Chlorophyta). Phytotaxa 372: 51.

Friedman J, Hastie T, and Tibshirani R. 2010. Regularization paths for generalized linear models via coordinate descent. J Stat Softw 33: 1-22.

Geisen S, Briones MJI, Gan H, et al. 2019. A methodological framework to embrace soil biodiversity. Soil Biol Biochem 136: 107536.

Gillespie MAK, Alfredsson M, Barrio IC, et al. 2020. Circumpolar terrestrial arthropod monitoring: A review of ongoing activities, opportunities and challenges, with a focus on spiders. Ambio 49: 704-17.

379 Martin M. 2011. Cutadapt removes adapter sequences from high-throughput sequencing reads. EMBnet.journal 17: 10.

381 Matsuoka K, Skoglund A, Roth G, et al. 2021. Quantarctica, an integrated mapping 
environment for Antarctica, the Southern Ocean, and sub-Antarctic islands. Environ Model Softw 140: 105015.

Muthukrishnan R and Rohini R. 2016. LASSO: A feature selection technique in predictive modeling for machine learning. In: 2016 IEEE International Conference on Advances in Computer Applications (ICACA). IEEE.

R Core Development Team. 2019. R: A language and environment for statistical computing. project.org/.

Siciliano SD, Palmer AS, Winsley T, et al. 2014. Soil fertility is associated with fungal and bacterial richness, whereas $\mathrm{pH}$ is associated with community composition in polar soil microbial communities. Soil Biol Biochem 78: 10-20.

Smykla J, Porazinska DL, Iakovenko NS, et al. 2018. Geochemical and biotic factors influencing the diversity and distribution of soil microfauna across ice-free coastal habitats in Victoria Land, Antarctica. Soil Biol Biochem 116: 265-76.

Thompson LR, Sanders JG, McDonald D, et al. 2017. A communal catalogue reveals Earth's multiscale microbial diversity. Nature 551: 457-63.

Tibshirani R. 1996. Regression shrinkage and selection via the Lasso. Society 58: 267-88.

Velasco-Castrillón A, Gibson JAE, and Stevens MI. 2014a. A review of current Antarctic

Velasco-Castrillón A, Schultz MB, Colombo F, et al. 2014b. Distribution and diversity of soil microfauna from East Antarctica: Assessing the link between biotic and abiotic

407 Wauchope HS, Shaw JD, and Terauds A. 2019. A snapshot of biodiversity protection in Antarctica. Nat Commun 10: 946.

409 Xin M and Zhou P. 2007. Mrakia psychrophila sp. nov., a new species isolated from $410 \quad$ Antarctic soil. J Zhejiang Univ Sci B 8: 260-5.

411 Zawierucha K, Marshall CJ, Wharton D, and Janko K. 2019. A nematode in the mist: 
412 Scottnema lindsayae is the only soil metazoan in remote antarctic deserts, at greater

413 densities with altitude. Polar Res 38: 1-12.

414 Zhang E, Thibaut LM, Terauds A, et al. 2020. Lifting the veil on arid-to-hyperarid Antarctic 415 soil microbiomes: a tale of two oases. Microbiome 8: 37.

\section{Figure Captions:}

418 Figure 1: Sampling area. All sampling locations are marked with a crosshair. Heat shading 419 (at map scale) indicates density of 18S Amplicon Sequence Variants (sensu Callahan et al. 420 2017) determined to be significantly influenced by substrate qualities as available. Base 421 layers compiled by the Norwegian Polar Institute and distributed in the Quantarctica package. 422 Visit http://www.quantarctica.org/. Base layers courtesy of the SCAR Antarctic Digital 423 Database, (C) 1993-2015 Scientific Committee on Antarctic Research; The National Snow and 424 Ice Data Centre, University of Colorado, Boulder; NASA, Visible Earth Team, 425 http://visibleearth.nasa.gov/; Australian Antarctic Division, (C) Commonwealth of Australia 4262006.

427 Figure 2: Counts of amplicon sequence variants for phyla deemed significantly influenced by 428 substrate composition (left) and examples of taxonomic assignments (right). The employed 429 relatively short primer pair resulted in survey data encompassing diverse soil life forms of 430 various phyla, at the expense of low-level taxonomic certainty, see Web Table 4 for 431 alignment qualities. (a) Mrakia frigida (Basidiomycota; prefect alignment) is closely related 432 to a recently described Antarctic species (Xin and Zhou 2007). (b) Chloroidium 433 angustoellipsoideum (Chlorophyta; perfect alignment) is in the same genus as the recently 434 described Chloroidium antarcticum (Darienko et al. 2018). (c) For Dileptus jonesi 435 (Ciliophora; 97.6\% identity) possible Antarctic distribution could not be confirmed. Both (d) 436 Scottnema lindsayae (Nematoda; perfect alignment) and (e) Mesobiotus furciger (Tardigrada; 437 perfect alignment) are known Antarctic species with good reference data coverage (Velasco438 Castrillón et al. 2014a). Base layers courtesy of the SCAR Antarctic Digital Database, (C) 439 1993-2015 Scientific Committee on Antarctic Research; The National Snow and Ice Data 440 Centre, University of Colorado, Boulder; NASA, Visible Earth Team, 441 http://visibleearth.nasa.gov/; Australian Antarctic Division, (C) Commonwealth of Australia 4422006. 
443 Table 1: Numerical summary of significant coefficient estimates for each phylum as obtained

444 through lasso logistic regression.

\begin{tabular}{|c|c|c|}
\hline 95\% CI Coefficient & $95 \%$ CI Odds ratio & $\begin{array}{l}\text { Proportion } \\
\text { of } \\
\text { bootstrap } \\
\text { replicates } \\
\text { not zero }\end{array}$ \\
\hline
\end{tabular}

\begin{tabular}{lllllll} 
phylum & predictor & lower & upper & lower & upper & \\
\hline Basidiomycota & Dolomite & 0 & 1.32 & 1 & -3.70 & 0.93 \\
& PH & -1.54 & 0.46 & 0.21 & -0.63 & 1.00 \\
Chlorophytes & MM* & -1.32 & -0.10 & 0.26 & 0.90 & 0.99 \\
& PH & -1.28 & -0.10 & 0.28 & 0.91 & 0.99 \\
Ciliophora & Garnets & -2.07 & -0.11 & 0.13 & 0.90 & 0.99 \\
& MM & -1.22 & 0.00 & 0.29 & 1.00 & 0.93 \\
& Sulphur & -3.14 & 0.00 & 0.04 & 1.00 & 0.85 \\
Nematodes & Cond & -2.17 & 0.00 & 0.11 & 1.00 & 0.99 \\
& MM & -2.10 & -0.26 & 0.12 & 0.77 & 0.99 \\
Tardigrades & PH & -1.42 & 0.00 & 0.23 & 1.00 & 0.95 \\
\hline
\end{tabular}


bioRxiv preprint doi: https://doi.org/10.1101/2021.08.18.456862; this version posted August 19, 2021. The copyright holder for this preprint (which was not certified by peer review) is the author/funder, who has granted bioRxiv a license to display the preprint in perpetuity. It is made available under aCC-BY-ND 4.0 International license.

445 


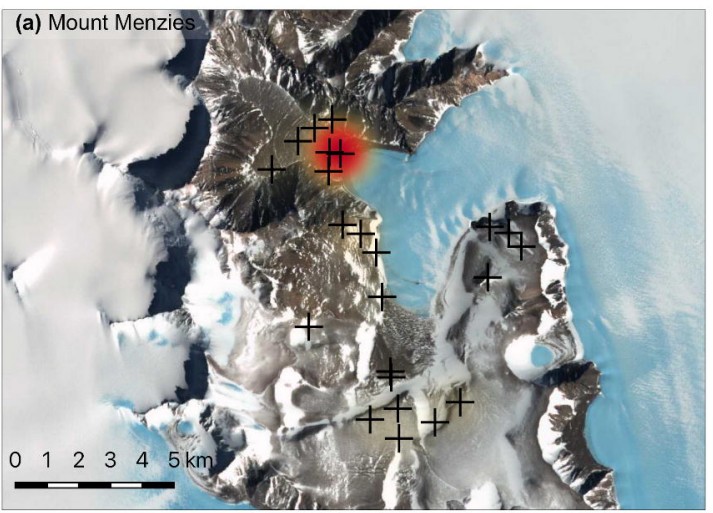

Overview Prince-Charles-Mountains
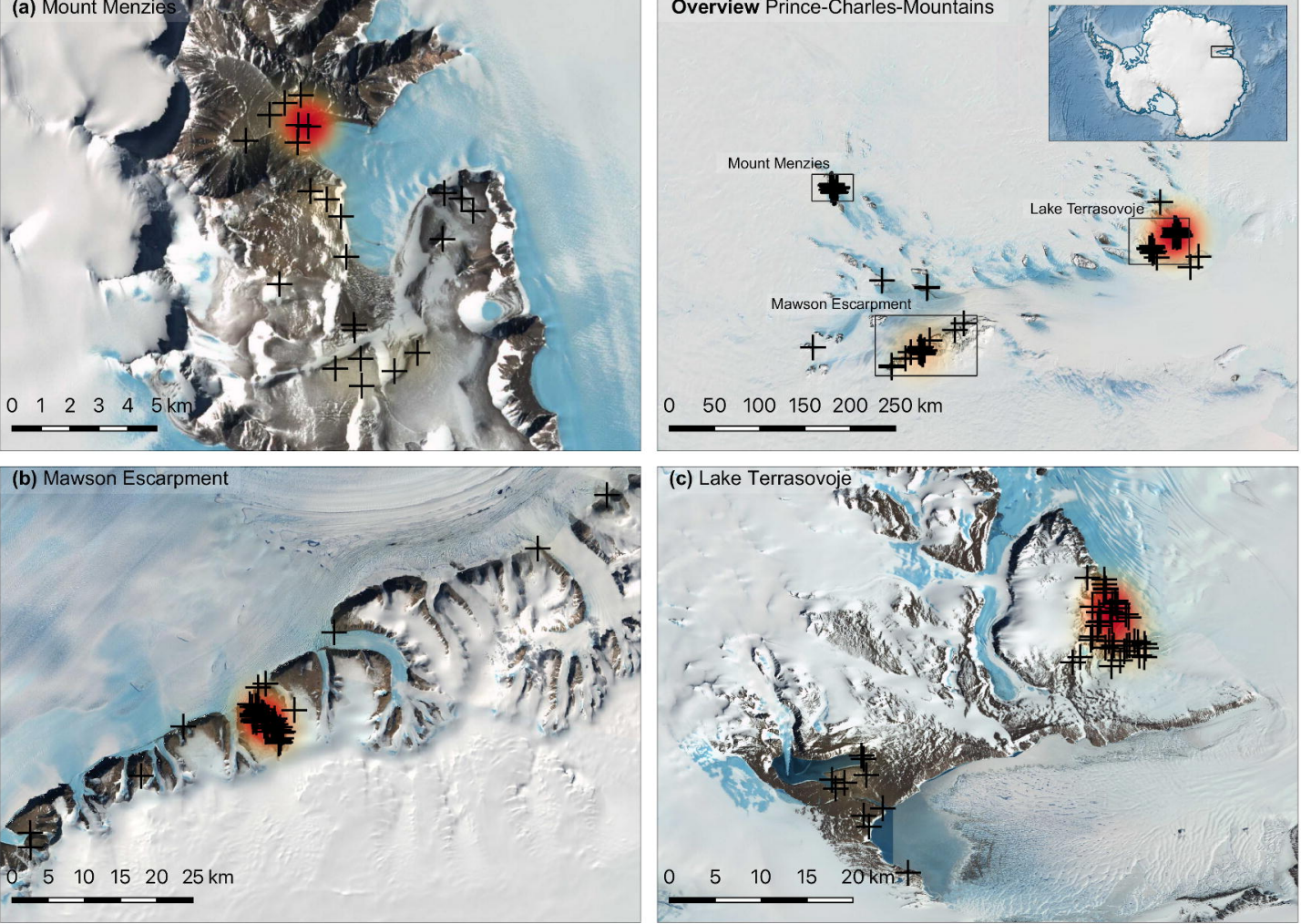


\section{(a) Basidiomycota}

\section{(2)}

8.

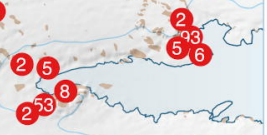

(b) Chlorophyta

0

Q3 3 (3)

$\because 8^{12}$ in mamrar

\section{(c) Ciliophora}

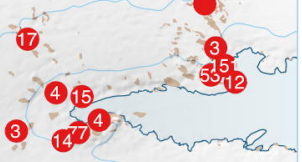

\section{(d) Nematoda}

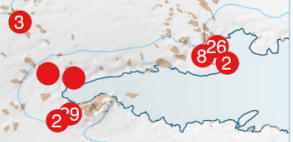

\section{(e) Tardigrada}

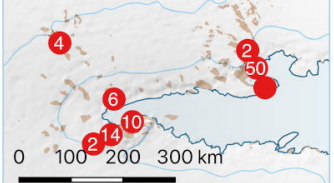

